\title{
Front Matter: Volume 10400
}

, "Front Matter: Volume 10400," Proc. SPIE 10400, Techniques and Instrumentation for Detection of Exoplanets VIII, 1040001 (15 December 2017); doi: $10.1117 / 12.2298430$

EDent: SPIE Optical Engineering + Applications, 2017, San Diego, California, SPIE. United States 


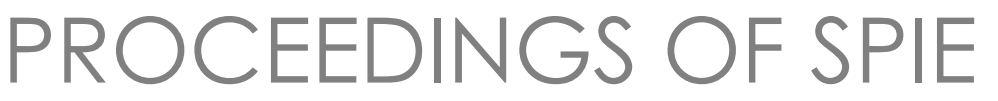

\title{
Techniques and Instrumentation for Detection of Exoplanets VIII
}

\author{
Stuart Shaklan \\ Editor
}

8-10 August 2017

San Diego, California, United States

Sponsored and Published by

SPIE 
The papers in this volume were part of the technical conference cited on the cover and title page. Papers were selected and subject to review by the editors and conference program committee. Some conference presentations may not be available for publication. Additional papers and presentation recordings may be available online in the SPIE Digital Library at SPIEDigitalLibrary.org.

The papers reflect the work and thoughts of the authors and are published herein as submitted. The publisher is not responsible for the validity of the information or for any outcomes resulting from reliance thereon.

Please use the following format to cite material from these proceedings:

Author(s), "Title of Paper," in Techniques and Instrumentation for Detection of Exoplanets VIII, edited by Stuart Shaklan, Proceedings of SPIE Vol. 10400 (SPIE, Bellingham, WA, 2017) Seven-digit Article CID Number.

ISSN: 0277-786X

ISSN: 1996-756X (electronic)

ISBN: 9781510612570

ISBN: 9781510612587 (electronic)

Published by

SPIE

P.O. Box 10, Bellingham, Washington 98227-0010 USA

Telephone +1 3606763290 (Pacific Time) · Fax +1 3606471445

SPIE.org

Copyright (c) 2017, Society of Photo-Optical Instrumentation Engineers.

Copying of material in this book for internal or personal use, or for the internal or personal use of specific clients, beyond the fair use provisions granted by the U.S. Copyright Law is authorized by SPIE subject to payment of copying fees. The Transactional Reporting Service base fee for this volume is $\$ 18.00$ per article (or portion thereof), which should be paid directly to the Copyright Clearance Center (CCC), 222 Rosewood Drive, Danvers, MA 01923. Payment may also be made electronically through CCC Online at copyright.com. Other copying for republication, resale, advertising or promotion, or any form of systematic or multiple reproduction of any material in this book is prohibited except with permission in writing from the publisher. The CCC fee code is 0277 $786 \mathrm{X} / 17 / \$ 18.00$.

Printed in the United States of America.

Publication of record for individual papers is online in the SPIE Digital Library.

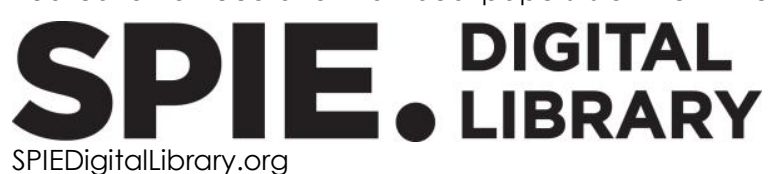

Paper Numbering: Proceedings of SPIE follow an e-First publication model. A unique citation identifier (CID) number is assigned to each article at the time of publication. Utilization of CIDs allows articles to be fully citable as soon as they are published online, and connects the same identifier to all online and print versions of the publication. SPIE uses a seven-digit CID article numbering system structured as follows:

- The first five digits correspond to the SPIE volume number.

- The last two digits indicate publication order within the volume using a Base 36 numbering system employing both numerals and letters. These two-number sets start with 00, 01, 02, 03, 04, 05, 06, 07, 08, 09, OA, OB ... 0Z, followed by 10-1Z, 20-2Z, etc. The CID Number appears on each page of the manuscript. 


\title{
Contents
}

\author{
ix Authors \\ xiii Conference Committee
}

\section{SESSION 1 WFIRST CORONAGRAPH I}

1040003 The WFIRST coronagraph instrument optical design update [10400-2]

1040004 WFIRST coronagraph optical modeling [10400-4]

1040005 Wavefront control performance modeling with WFIRST shaped pupil coronagraph testbed [10400-5]

1040006 Sensitivity of WFIRST coronagraph broadband contrast performance to DM actuator errors [10400-6]

SESSION 2 WFIRST CORONAGRAPH II

1040007 Sensitivity of the WFIRST coronagraph performance to key instrument parameters [10400-7]

1040009 Current science requirements and planned implementation for the WFIRST-CGI Integral Field Spectrograph (IFS) [10400-9]

10400 OB Simulating the WFIRST coronagraph Integral Field Spectrograph [10400-11]

\section{SESSION $3 \quad$ WFIRST CORONAGRAPH III}

10400 OC Fabrication of coronagraph masks and laboratory scale star-shade masks: characteristics, defects, and performance [10400-12]

10400 OD Dynamic testbed demonstration of WFIRST coronagraph Low Order Wavefront Sensing and Control (LOWFS/C) [10400-13]

10400 OE Shaped pupil coronagraphy for WFIRST: high-contrast broadband testbed demonstration [10400-14]

10400 OF Hybrid Lyot coronagraph for WFIRST: high-contrast broadband testbed demonstration [10400-15]

\section{SESSION 4 SEGMENTED APERTURE CORONAGRAPHS}

10400 OG Capabilities of ACAD-OSM, an active method for the correction of aperture discontinuities [10400-16] 
$10400 \mathrm{OH} \quad$ Coronagraph design optimization for segmented aperture telescopes [10400-17]

10400 0J Performance and sensitivity of vortex coronagraphs on segmented space telescopes [10400-19]

10400 OM Sensitivity analysis for high-contrast missions with segmented telescopes [10400-22]

10400 ON Design, fabrication, and testing of stellar coronagraphs for exoplanet imaging [10400-64]

1040000 Shaped pupil coronagraph design improvements for the WFIRST Coronagraph Instrument [10400-73]

\section{SESSION 5 CORONAGRAPH DESIGN, COMPONENTS, AND ANALYSIS I}

10400 OP Electric field conjugation in the presence of model uncertainty [10400-23]

$10400 \mathrm{OQ}$ Wavefront control methods for high-contrast integral field spectroscopy [10400-24]

10400 OR Improved high-contrast wavefront controllers for exoplanet coronagraphic imaging systems [10400-25]

10400 OS Adaptive estimation of line-of-sight jitter disturbance [10400-26]

10400 OT Tip/tilt optimizations for polynomial apodized vortex coronagraphs on obscured telescope pupils [10400-27]

10400 OU Patterned liquid-crystal optics for broadband coronagraphy and wavefront sensing [10400-28]

\section{SESSION 6 CORONAGRAPH DESIGN, COMPONENTS, AND ANALYSIS II}

10400 OV Optimal design of apodizing phase plate coronagraphs [10400-29]

10400 OW High contrast imaging in multi-star systems: progress in technology development and lab results [10400-30]

10400 oY Utilizing active single-mode fiber injection for speckle nulling in exoplanet characterization [10400-32]

$10400 \mathrm{OZ}$ Baseline requirements for detecting biosignatures with the HabEx and LUVOIR mission concepts [10400-33]

1040010 Optical tolerances for the PICTURE-C mission: error budget for electric field conjugation, beam walk, surface scatter, and polarization aberration [10400-34] 
1040011 Characterization of low mass deformable mirrors and ASIC drivers for high-contrast imaging [10400-35]

1040013 Design of the deformable mirror demonstration CubeSat (DeMi) [10400-37]

1040014 Fundamental limits to high-contrast wavefront control [10400-80]

SESSION 8 GROUND-BASED INSTRUMENTS

1040015 Combining angular differential imaging and accurate polarimetry with SPHERE/IRDIS to characterize young giant exoplanets [10400-38]

1040016 First light of the CHARIS high-contrast integral-field spectrograph [10400-39]

1040018 NIRPS: an adaptive-optics assisted radial velocity spectrograph to chase exoplanets around M-stars [10400-41]

\section{SESSION 9 STARSHADES}

1040019 Modeling and performance predictions for the Princeton Starshade Testbed [10400-42]

104001 A Optical demonstration of a starshade at flight Fresnel numbers [10400-43]

10400 lB Precise starshade stationkeeping and pointing with a Zernike wavefront sensor [10400-44]

10400 1C Starshade mechanical design for the Habitable Exoplanet imaging mission concept (HabEx) [10400-45]

10400 1D Prospects for exoplanet imaging in multi-star systems with starshades [10400-46]

\section{SESSION 10 ASTROMETRY}

10400 1E Gaia and exoplanets: a revolution in the making (Invited Paper) [10400-47]

$104001 G$ Results of the astrometry and direct imaging testbed for exoplanet detection [10400-49]

SESSION 11 INTERFEROMETRIC CORONAGRAPHY

1040011 Segmented Aperture Interferometric Nulling Testbed (SAINT) II: component systems update [10400-51]

$104001 \mathrm{~J}$ Phase-shifting coronagraph [10400-59] 


$\begin{aligned} & \text { SESSION } 12 \text { MISSION AND DATA ANALYSIS } \\ & 104001 \mathrm{~K} \begin{array}{l}\text { ExEP yield modeling tool and validation test results [10400-53] } \\ 104001 \mathrm{~L}\end{array} \\ & \begin{array}{l}\text { Multi-mission modeling for space-based exoplanet imagers [10400-54] } \\ 104001 \mathrm{~N}\end{array} \quad \begin{array}{l}\text { Quantifying the impact of small statistics on planet detectability at small inner working } \\ \text { angles [10400-56] }\end{array} \\ & \begin{array}{l}\text { Planet signal extraction from direct imaging using common spatial pattern filtering } \\ \text { [10400-57] }\end{array} \\ & \text { POSTERS SESSION }\end{aligned}$

$104001 \mathrm{P}$ Commissioning and performance results of the WFIRST/PISCES integral field spectrograph [10400-58]

$104001 Q$ Development status and performance of the evanescent wave coronagraph testbed [10400-60]

10400 is Simulation of realistic images for Starshade missions [10400-62]

10400 1T Shape accuracy requirements on starshades for large and small apertures [10400-63]

$104001 \mathrm{~S}$ Starshade orbital maneuver study for WFIRST [10400-65]

$104001 \mathrm{~V}$ Line profile analysis of the laser frequency comb in FOCES [10400-66]

$104001 \mathrm{X}$ Optimization of high-inclination orbits using planetary flybys for a zodiacal light-imaging mission [10400-68]

$104001 Y \quad$ Detected exoplanet population distributions found analytically [10400-69]

1040012 The low-order wavefront control system for the PICTURE-C mission: preliminary testbed results from the Shack-Hartmann sensor [10400-70]

1040020 The low-order wavefront control system for the PICTURE-C mission: high-speed image acquisition and processing [10400-71]

1040021 Post-processing of the HST STIS coronagraphic observations [10400-72]

1040022 Optimizing the regularization in broadband wavefront control algorithm for WFIRST coronagraph [10400-74]

1040024 Focal plane based wavefront sensing with random DM probes [10400-76]

1040026 The automated data processing architecture for the GPI Exoplanet Survey [10400-78]

1040027 Optimization of pyKLIP's forward model matched filter for the GPI Exoplanet Survey [10400-79] 
1040028 Identification of the focal plane wavefront control system using E-M algorithm [10400-81]

1040029 A fiber injection unit for the Keck Planet Imager and Characterizer [10400-82]

Proc. of SPIE Vol. $104001040001-7$

Downloaded From: https://www.spiedigitallibrary.org/conference-proceedings-of-spie on 26 Apr 2023 Terms of Use: https://www.spiedigitallibrary.org/terms-of-use 
Proc. of SPIE Vol. $104001040001-8$

Downloaded From: https://www.spiedigitallibrary.org/conference-proceedings-of-spie on 26 Apr 2023 Terms of Use: https://www.spiedigitallibrary.org/terms-of-use 


\section{Authors}

Numbers in the index correspond to the last two digits of the seven-digit citation identifier (CID) article numbering system used in Proceedings of SPIE. The first five digits reflect the volume number. Base 36 numbering is employed for the last two digits and indicates the order of articles within the volume. Numbers start with 00, 01, 02, 03, 04, 05, 06, 07, 08, 09, OA, OB...0Z, followed by 10-1Z, 20-2Z, etc.

Agolli, Jack, 11 Alagao, Mary Angelie, 1Q

Allan, Gregory, 13

Amiri, Nikta, 04

An, Xin, OD

Arriaga, Pauline, 26

Artigau, E., 18

Arya, Manan, $1 \mathrm{C}$

Balasubramanian, Kunjithapatham, OC, OD, OE

Bandy, T., 18

Barnes, Derek, 13

Bartos, R. D., 29

Belikov, Ruslan, OW, 1D, 1G, 1N, 24

Bendek, Eduardo A., OW, 1D, 1G, 24

Bender, Ralf, 1V

Benz, W., 18

Beuzit, Jean-LuC, 15

Blind, N., 18

Boisse, I., 18

Bolcar, Matthew R., 11

Bottom, Michael, 1B

Bouchy, F., 18

Bovay, S., 18

Bradford, S. Case, $1 \mathrm{C}$

Brandt, Timothy, OB, 16

Brewer, John, ON

Broeg, Ch., 18

Brousseau, D., 18

Bryson, Stephen T., $1 \mathrm{~N}$

Buisset, Christophe, 1Q

Burke, Elliot, 11

Cabral, A., 18

Cady, Eric J., 05, OB, OC, OD, OE, OF, OQ, 1B, 1P, IT

Cahoy, Kerri L., OB, 13

Carlotti, Alexis, $1 \mathrm{~J}$

Chakrabarti, Supriya, 10, 12, 20

Chilcote, Jeffrey K., 16, 26

Choquet, Élodie, OY, 21

Conod, U., 18

Cook, Timothy A., 10, 1Z, 20

Corsetti, James, 11

Currie, Thayne, 16

Debes, John H., 21

de Boer, Jozua, 15

Dekany, R., OY

de Kok, Remco J., 15

Delabre, B., 18

Delacroix, Christian, 1K, 1L, 1U

Delfosse, X., 18
Delorme, Jacques-Robert, OY, OZ, 29

de Medeiros, J. R., 18

Demers, Richard, 03

De Rosa, Robert J., 26

DeVries, John, 11

Dillon, Thomas, 11

Doelman, David S., OU

Douglas, Ewan S., OB, 13

Doyon, R., 18

Dudinov, Vladimir N., 24

Dumont, Philip, 19

Eberhardt, Andrew, 11

Echeverri, D., OY

Echternach, Pierre, OC

Egron, Sylvain, OG

Eldorado Riggs, A. J., 00

Escuti, Michael J., OU

Figueira, P., 18

Figura, Joseph S., 13

Finan, Emily, $1 \mathrm{G}$

Finn, Susanna C., 10, 1Z, 20

Fitzgerald, M., 29

Fogarty, Kevin, 0G, OT

Freebury, Gregg, 1C

Fregoso, Santos, OC

Fucik, J., OY

Fusco, Thierry, OM

Galvin, Michael, 16, 1A

Garrett, Daniel, 1K, 1L, IU, IY

Genolet, L., 18

Gersh-Range, Jessica, OE

Ginski, C., 15

Girard, Julien H., 15

Gong, Qian, 09, 0B, 0Q, 1P

González Hernández, J. I., 18

Gordon, Brian, OF

Graham, James R., 26

Groff, Tyler D., 09, 0B, 0Q, 0R, 16, 1P

Grogan, Keith, IX

Grupp, Frank, IV

Gubner, Jennifer N., 13

Gull, Jamie, 1C

Gutt, Gary, 04

Guyon, Olivier, ON, 0Q, 16, 1G

Hagelberg, J., 18

Hamilton, Ryan, ON

Harness, Anthony D., 19, 1A, 1S

Haughwout, Christian A., 13

Hayashi, Masahiko, 16 
Helmbrecht, Michael A., 11

Hénault, François, $1 \mathrm{~J}$

Hernandez, O., 18

Hewawasam, Kuravi, 10, 12, 20

Hicks, Brian A., 11

Hilgemann, Evan, 1C

Holte, Elias, ID

Hopp, Ulrich, $1 \mathrm{~V}$

Howe, Glenn A., 10, 1Z, 20

Hu, Mengya (Mia), 1A, is

$\mathrm{Hu}$, Renyu, $\mathrm{OZ}$

Jewell, Jeffrey, $\mathrm{OH}, \mathrm{O}$

Johnson, Lee, $1 \mathrm{G}$

Jovanovic, Nemanja, 16, 29

Kasdin, N. Jeremy, OE, OR, OS, 16, 19, 1A, 1S, 28

Kasper, Markus, 15

Käufl, U., 18

Keller, Christoph U., 15

Kellermann, Hanna, $1 \mathrm{~V}$

Kern, Brian, 05, 06, OD, OE, OF, OP, 22

Kim, Yunjong, 19, 1A, 1S

Klimovich, Nikita, OY, 0Z, 29

Knapp, Gillian, 16

Knight, Justin M., ON, $1 \mathrm{G}$

Knoedler, Alex A., 13

Krist, John E., 03, 04, 05, 07, OB, 00

Lam, Raymond, OD, OE, OF

Langlois, Maud, 15

Lea, Andrew, 11

Leboulleux, Lucie, 0G, OM

LeClair, Sarah, 13

Lepine, Thierry, 1Q

Lilley, S., 29

Lisman, P. Douglas, 1C

Liu, Xiang Cate, $1 \mathrm{~K}$

Llop Sayson, Jorge, IP

Lloyd, James, $1 \mathrm{X}$

Loomis, Craig, 16

Lowrance, Patrick, $1 \mathrm{~K}$

Lozi, Julien, 16

Lupu, Roxana, 09

Macintosh, Bruce, 10, 26, 27

Malo, L., 18

Mandell, Avi M., 09, OB, 0Q, IP

Marchen, Luis, 04, $1 T$

Marois, Christian, 26

Martel, Jason, 10, 1Z, 20

Martin, Stefan, 1B

Marx, David, 04, OD, OF, OP, 22

Mawet, Dimitri, OH, OJ, OY, OZ, 29

Mazoyer, Johan, 0G, 0T, 14

McCabe, Tyler, 11

McElwain, Michael W., 09, OB, OQ, 1P

McGown, James, $1 C$

McGuire, James, 03, 04

Mejia Prada, Camilo, OB, OC, OD, OE, OF, OQ, 11 , $1 \mathrm{P}$

Melo, C., 18

Mendillo, Christopher B., 10, 1Z, 20

Mennesson, Bertrand, 07, OY
Merck, John, 13

Metzman, Jacob, OC

Millar-Blanchaer, Maxwell A., 26

Milster, Thomas, ON, $1 \mathrm{G}$

Moody, Dwight, OD, OE, OF

Morgan, Rhonda, $1 \mathrm{~K}$

Mouillet, David, 15

Muller, Richard, OC, OF

Murphy, Thomas J., 13

N'Diaye, Mamadou, OG, OM, OT

Nemati, Bijian, 04, 05, 06, 07, 00, 0P, 22

Norman, Colin, OG

Nunez, Paul, 1K

Opperman, Roedolph A., 13

Patterson, Keith, OD, OE, OF

Pellicori, Samuel, 11

Pepe, F., 18

Perrin, Marshall D., 21, 26

Petrone, Peter, III, 11

Pluzhnik, Eugene, OW, 1G, 24

Poberezhskiy, llya, 05, 06, 0D, OE, OF, OP, 22

Por, Emiel H., OV

Poshyachinda, Saran, $1 Q$

Pueyo, Laurent, OG, OM, OT, 14, 21, 27

Rabbia, Yves, $1 Q$

Rameau, Julien, 26

Randolph, M., OY

Ranganathan, Nikhil, 10

Rasilla, J. L., 18

Rebolo, R., 18

Redding, Dave, $\mathrm{OH}$

Ren, Bin, 21

Reshetov, V., 18

Riggs, A. J. Eldorado, O4, OB, OC, OE, OR, ID

Rizzo, Maxime Jean, 09, OB, 0Q, 16, 1P, 1S

Roberge, Aki, OB, is

Roberts, Lewis C., Jr., 11

Rodack, Alexander, $1 \mathrm{G}$

Ruane, Garreth, OH, OJ, OY, OZ, 29

Rud, Mayer, 03

Ruffio, Jean-Baptiste, 10, 26, 27

Ryan, Daniel, OC

Saddlemyer, L., 18

Saini, Navtej, 04

Santos, N. C., 18

Sarajlic, M., 18

Sauvage, Jean-François, $0 M$

Savransky, Dmitry, 1K, 1L, 10, 1U, 1X, 1Y, 26

Saxena, Prabal, 09, OB, 0Q, IP

Segovilla, A., 18

Seo, Byoung-Joon, 06, OC, OD, OE, OF, OP, 22

Serabyn, E., OY

Seubert, Carl, 1B

Shaklan, Stuart B., OH, 0J, 19, 1A, 1B, 1C, 1D, 1T

Shapiro, Jacob, 10, 26

Sheets, Teresa, 11

Shelton, Chris, 11

Shi, Fang, OC, OD, OE, OF

Shields, Joel, OD

Shiri, Ron, 11 
Sidick, Erkin, 04, 06, OD, OF, OP, 22

Sinha, Amlan, 1U, $1 \mathrm{X}$

Sirbu, Dan, OW, 1A, 1D, 24

Skouloudis, Nikolaos, 13

Snik, Frans, OU, 15

Soonthornthum, Boonrucksar, 1Q

Sordet, M., 18

Soto, Gabriel, 1U, 1X

Soummer, Rémi, OM

Sozzetti, Alessandro, $1 \mathrm{E}$

St. Laurent, Kathryn, OM

Stam, Daphne M., 15

Steeves, John, 1C

Subedi, Hari, OS

Sun, He, OR, 28

Takato, Naruhisa, 16

Tang, Hong, 03, 04, OD, OE

Thibault, S., 18

Thomson, Mark, $1 \mathrm{C}$

Trauger, John T., 06, OD, OE, OF

Trease, Brian, 1C

Truong, Tuan, OD

Turmon, Michael, $1 \mathrm{~K}$

Vallée, Ph., 18

van Holstein, Rob G., 15

Vanderbei, Robert J., OR, 1A, 1S, 28

Varnai, Peter, OS

Vasisht, G., OY

Vérinaud, Christophe, $1 \mathrm{~J}$

Vigan, Arthur, 15

Wallace, J. K., OY, 29

Wang, Jason J., 26, 27

Wang, Ji, OY, OZ, 29

Wang, Liang, IV

Ward, Karen, ON

Warriner, Nathaniel Z., OU

Warwick, Steve, 1C

Webb, David, $1 \mathrm{C}$

White, Victor, OC, OD

Wildi, F., 18

Wilson, Daniel, OC, OD, OF

Wilson, Robert Casey, OC

Wizinowich, P. L., 29

Wolff, Schuyler G., 26

Wu, Xingtao, 11

Wu, Yuqian, 11

Xin, Y., OY, 29

Xuan, W., OY

Yao, Li, 11

Yee, Karl, OC

Zareh, Shannon Kian, 1B

Zhao, Feng, 03

Zhou, Hanying, 04, 05, OD, OE

Zimmermann, Neil T., 09, OB, OE, 0O, OQ

Zurlo, Alice, 15 
Proc. of SPIE Vol. 10400 1040001-12 Downloaded From: https://www.spiedigitallibrary.org/conference-proceedings-of-spie on 26 Apr 2023
Terms of Use: https://www.spiedigitallibrary.org/terms-of-use 


\title{
Conference Committee
}

\author{
Conference Chair
}

Stuart Shaklan, Jet Propulsion Laboratory (United States)

Program Track Chair

Oswald H. Siegmund, University of California, Berkeley (United States)

Conference Program Committee

Olivier Guyon, Subaru Telescope, National Astronomical Observatory of Japan (United States) and Research Corporation of University of Hawaii (United States) and The University of Arizona (United States)

Lucas Labadie, University of Cologne (Germany)

Bruce A. Macintosh, Stanford University (United States)

Dimitri P. Mawet, California Institute of Technology (United States)

M. Charley Noecker, Jet Propulsion Laboratory (United States)

Rémi Soummer, Space Telescope Science Institute (United States)

\section{Conference Review Committee}

Howard A. MacEwen, Reviresco LLC (United States)

James Breckinridge, California Institute of Technology (United States)

Tony B. Hull, The University of New Mexico (United States)

Dae Wook Kim, College of Optical Sciences, The University of Arizona (United States)

Pascal Hallibert, European Space Research and Technology Center (Netherlands)

\section{Session Chairs}

1 WFIRST Coronagraph I

Stuart Shaklan, Jet Propulsion Laboratory (United States)

2 WFIRST Coronagraph II

Garreth Ruane, California Institute of Technology (United States)

3 WFIRST Coronagraph III

Stuart Shaklan, Jet Propulsion Laboratory (United States)

4 Segmented Aperture Coronagraphs

Eric Cady, Jet Propulsion Laboratory (United States) 
5 Coronagraph Design, Components, and Analysis I Lucas Labadie, Universität zu Köln (Germany)

6 Coronagraph Design, Components, and Analysis II

A. J. Eldorado Riggs, Jet Propulsion Laboratory (United States)

7 Deformable Mirrors

Garreth Ruane, California Institute of Technology (United States)

8 Ground-based Instruments

Michael Bottom, Jet Propulsion Laboratory (United States)

9 Starshades

Stuart Shaklan, Jet Propulsion Laboratory (United States)

10 Astrometry

A. J. Eldorado Riggs, Jet Propulsion Laboratory (United States)

11 Interferometric Coronagraphy

A. J. Eldorado Riggs, Jet Propulsion Laboratory (United States)

12 Mission and Data Analysis

Lucas Labadie, Universität zu Köln (Germany) 